\title{
Validation of a Sensitivity Performance Index Test Protocol and Evaluation of Colour Doppler Sensitivity for a Range of Ultrasound Scanners
}

\author{
Jacinta Browne \\ Technological University Dublin, jacinta.browne@tudublin.ie \\ Amanda Watson \\ Western Infirmary Glasgow \\ Peter Hoskins \\ University of Edinburgh
}

See next page for additional authors

Follow this and additional works at: https://arrow.tudublin.ie/scschphyart

Part of the Physics Commons

\section{Recommended Citation}

Browne, J., Watson, A., Hoskins, P., Elliott, A.: Validation of a Sensitivity Performance Index Test Protocol and Evaluation of Colour Doppler Sensitivity for a Range of Ultrasound Scanners. Ultrasound in Medicine and Biology, Vol.31 (7), pp. 1475-1483. 2004. doi:10.1016/j.ultrasmedbio.2004.09.005

This Article is brought to you for free and open access by the School of Physics \& Clinical \& Optometric Science at ARROW@TU Dublin. It has been accepted for inclusion in Articles by an authorized administrator of ARROW@TU Dublin. For more information, please contact arrow.admin@tudublin.ie, aisling.coyne@tudublin.ie, gerard.connolly@tudublin.ie.

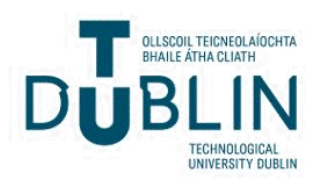


Authors

Jacinta Browne, Amanda Watson, Peter Hoskins, and Alex Elliott

This article is available at ARROW@TU Dublin: https://arrow.tudublin.ie/scschphyart/11 
VALIDATION OF A SENSITIVITY PERFORMANCE INDEX TEST PROTOCOL AND EVALUATION OF COLOUR DOPPLER SENSITIVITY FOR A RANGE OF ULTRASOUND SCANNERS

JACINTA E BROWNE ${ }^{1}$, AMANDA J WATSON ${ }^{1}$, PETER R HOSKINS ${ }^{2}$ AND ALEX T ELLIOTT ${ }^{1}$

Department of Clinical Physics and Bioengineering, Western Infirmary Glasgow, Glasgow, UK ${ }^{1}$;

Medical Physics Unit, University of Edinburgh, Edinburgh, $\mathrm{UK}^{2}$.

Dr Jacinta Browne, Department of Nuclear Cardiology, Queen Elizabeth Building, Glasgow Royal Infirmary, 16 Alexandra Parade, Glasgow, G31 2ER, UK.

Tel: $0141-2110501$

Fax: 0141-2111252

Email: Jacinta.Browne@northglasgow.scot.nhs.uk

Running Title: Sensitivity Performance Index

Key Words: Colour Doppler Sensitivity; Doppler Flow Phantom; Sensitivity Performance Index; Penetration Depth; Lowest Detectable Velocity; Instrument Settings. 


\section{VALIDATION OF A SENSITIVITY PERFORMANCE INDEX TEST \\ PROTOCOL AND EVALUATION OF COLOUR DOPPLER SENSITIVITY \\ FOR A RANGE OF ULTRASOUND SCANNERS}

\section{ABSTRACT:}

The ability to detect flow is the most crucial aspect of an ultrasound system since, if flow cannot be detected, no other aspect of performance matters. The objectives of this study were to validate a Doppler "sensitivity performance index", a figure of merit, and to determine whether it could be used to differentiate colour Doppler sensitivity performance in scanners of varying complexity. The sensitivity performance index was developed to give a combined measure of related aspects of sensitivity, such as the lowest detectable velocity, the vessel size and the penetration depth. The colour Doppler sensitivity was evaluated objectively as the lowest detectable velocity signal from the deepest achievable point within the Doppler sensitivity phantom free from extraneous noise in a small diameter vessel $(3.2 \mathrm{~mm}$ inner diameter). The effect of vessel size and mean velocity on the sensitivity performance index were investigated and it was found that the index was not proportional to vessel size, but this may be accounted for by considering the effect of the acoustic properties of the vessel material, the clutter filter and beam shape. The results obtained using flow phantoms with vessel sizes different to those used in this study are therefore, not directly comparable with the results found in this study; however, a similar trend should be found in the results for the effect of control settings and a similar range of ultrasound scanners. It was found that the Doppler sensitivity performance index was a robust challenging test, as none of the ultrasound scanners evaluated was capable of achieving the highest sensitivity performance index score, which would be limited by the lowest pump velocity and the deepest point of 
the vessel within the flow phantom. Therefore, this suggests that this method of determining Doppler sensitivity performance is valuable in the absence of other suitable methods, despite the fact that the relationship between the sensitivity performance index and vessel size is not proportional. Furthermore, use of the Doppler sensitivity performance index for the evaluation of a range of scanners demonstrated that curvilinear transducers have higher sensitivity performance indices than higher frequency linear transducers, due to the higher achievable penetration depth. The effect of instrument settings was assessed for two transducers, the 4C3 curvilinear general-purpose transducer (Aspen) and the PVM375AT curvilinear general-purpose transducer (Nemio). The colour Doppler sensitivity performance was found to be significantly dependent on the clutter filter setting and the output power setting for both transducers tested. Users need to be aware of the effect of these settings on the colour Doppler sensitivity performance of their ultrasound scanner when interpreting the clinical significance of the colour Doppler information. (Email: Jacinta.Browne@northglasgow.scot.nhs.uk)

Key Words: Colour Doppler Sensitivity; Doppler Flow Phantom; Sensitivity Performance Index; Penetration Depth; Lowest Detectable Velocity; Instrument Settings. 


\section{INTRODUCTION}

Doppler sensitivity is an important aspect of Doppler performance, since, in clinical examinations, the question often arises as to whether blood flow is present, for example: to distinguish between trickle blood flow (low velocity) and no blood flow in the case of the evaluation of carotid artery stenosis (Mattos et al 1992); to verify parenchymal perfusion by colour Doppler post-renal-transplant (Allan et al 2000); or examine the venous system of the lower limb for venous insufficiency or thrombosis (Baxter 1997). Therefore, sensitivity is one of the most critical aspects of colour and power Doppler performance since, if blood flow cannot be detected, no other aspect of performance matters. Doppler sensitivity of ultrasound scanners is defined in this study as a measure of the minimum signal strength (from different diameter vessels and from different depths within the body) for the lowest detectable velocity that can be detected unambiguously in the absence of an interference signal (AIUM 1993; Wang et al 1992). Doppler sensitivity should not be confused with a Doppler system's detectability, which is the system's ability to distinguish a small echo signal from an interference signal (AIUM 1993). The interference signal detected by the Doppler system is made up of system noise and clutter from the movement of blood vessel walls and slow-moving or stationary solid tissue within the sample volume. Doppler sensitivity is related to two factors: these are the detection of low velocity blood flow $\left(<10 \mathrm{~cm} \mathrm{~s}^{-1}\right)$ and the presence of a few moving scatterers. Therefore, both lowered Doppler frequency shift and decreased Doppler signal strength contribute to the measured sensitivity. The Doppler sensitivity was defined as a measure of the maximum depth of a vessel in tissue from which a Doppler system could detect a signal free from extraneous noise (Hoskins et al 1994). 
Attempts have been made to evaluate spectral Doppler sensitivity by Wang et al (1992) using a vibrating disk, which allowed the frequency output and the signal amplitude to be controlled precisely. Using this method, the authors were able to generate a threshold curve which reflected the minimum detectable signal over the frequency range of interest. However, this method was unable to evaluate colour Doppler sensitivity, due to the bidirectional symmetrical side-band ambiguity produced by the test phantom. Rickey and Fenster (1996), attempted to evaluate both spectral and colour Doppler detectability in the presence of a controllable clutter signal. However, this method was time-consuming and complex and involved the generation of a 4-D dataset. Furthermore, this test phantom was difficult to build; to date, is the only such test phantom available and is not suitable for performing routine quality control tests in a busy department. Therefore, an alternative simplified method was needed to determine Doppler sensitivity performance. The aim of this paper is to introduce and evaluate a Doppler sensitivity performance index, a figure of merit, for simple and rapid evaluation of Doppler sensitivity performance in a busy ultrasound department. The Doppler sensitivity performance index is a potentially valuable quantity for describing Doppler ultrasound performance, because it combines key Doppler parameters into a single figure of merit. 


\section{METHODS}

\section{Sensitivity performance index}

Colour Doppler sensitivity was determined using a sensitivity performance index, which used the same criteria as those of Wang et al (1992). These criteria were that Doppler sensitivity can be simplified to two questions: what is the lowest frequency or velocity that can be detected and, for a given frequency or velocity, how much signal strength (determined by vessel size and vessel depth) is required for the signal to be detected? The sensitivity performance index used in this study combined penetration depth, lowest detectable velocity and vessel size into a single figure of merit given in eqn (1). The premise of the index being, the greater the penetration depth and the lower the detected velocity, then the greater the sensitivity performance index score for the ultrasound scanner.

Sensitivity performance index $=\left(\mathrm{P}_{\mathrm{LV}}\right) /(\mathrm{D} \times \mathrm{LV})$

where: $\mathrm{P}_{\mathrm{LV}}$ is the penetration depth of lowest detectable velocity, which is the maximum depth in tissue from which a Doppler signal free from extraneous noise can be obtained; D is the vessel diameter from which the Doppler signal is detected; and $\mathrm{LV}$ is the lowest detectable velocity, which is the lowest velocity that can be displayed unambiguously. Use of the diameter parameter, D, was included to allow the index to be used for different vessel sizes, so that the technique may be used in alternative flow systems.

\section{Penetration Depth Flow Phantom}

A penetration depth flow phantom (Fig. 1) with three different sized vessels $(1.6 \mathrm{~mm}$, $3.2 \mathrm{~mm}$ and $4.8 \mathrm{~mm}$ ) was used. The flow phantom consisted of a block of 
tissue-mimicking material surrounding the vessel, which was orientated at an angle of $60^{\circ}$ to the ultrasound beam, and whose maximum depth was $160 \mathrm{~mm}$. A blood-mimicking fluid (BMF), the physical and acoustic properties of which are listed in Table 1 (Ramnarine et al 1998; Ramnarine et al 1999), was pumped through the vessel. The flow phantom was filled by Gammex-RMI with a water-based gel TMM, with powdered graphite added to provide attenuation. This TMM was reported by Gammex-RMI to have an attenuation coefficient and speed of sound of $0.7 \mathrm{~dB} \mathrm{~cm}^{-1} \mathrm{MHz}^{-1}$ and $1540 \mathrm{~m} \mathrm{~s}^{-1}$, respectively, at $22{ }^{\circ} \mathrm{C}$. The vessels were made from C-flex tubing (Cole-Parmer, Walden, UK), which has been reported to have an attenuation coefficient and speed of sound of $5.6 \mathrm{~dB} \mathrm{~cm}^{-1} \mathrm{MHz}^{-1}$ and $1553 \mathrm{~m} \mathrm{~s}^{-1}$, respectively, at $22{ }^{\circ} \mathrm{C}$ (Hoskins et al 1994).

A computer-controller system was used to send control signals (steady state) to the micropump (CP 07002-25: Cole-Parmer, Walden, UK). A multifunction I/O board (National Instruments, TX, USA) was used to output the voltage signal from the computer to the motor.

\section{Ultrasound scanners}

The colour and power Doppler modes for a selection of the curvilinear and linear transducers of the following 10 ultrasound scanners were evaluated in this study: an ATL HDI 5000 (ATL / Philips, Amsterdam, The Netherlands), an Acuson Aspen (Acuson/Siemens, CA, USA), an Acuson 128 (Acuson/Siemens, CA, USA), a SonoSite 180 (SonoSite, WA, USA), a SonoSite 180Plus (SonoSite, WA, USA), a Siemens Sienna (Siemens Medical Solutions, Karlsruhe, Germany), an Aloka SSD 
5000 (Aloka Ltd, Tokyo, Japan), a Toshiba Aplio (Toshiba Medical Systems, Japan), a Toshiba Nemio (Toshiba Medical Systems, Japan); and a Siemens Antares (Siemens Medical Solutions, Karlsruhe, Germany). A summary of the transducers and modes tested for each of the ultrasound scanners is presented in Table 4. The ultrasound scanners were chosen to represent a cross-section of scanners, with regard to age (ranging from about $10 \mathrm{y}$ to just a few months in age) and cost (Table 4).

\section{Sensitivity performance index test protocol}

The flow phantom's scanning-well was filled with speed-of-sound-corrected water (water 90.5\%: propanol 9.5\%) (Martin and Spinks 2001) and the transducer was positioned longitudinally over the vessel of interest by using a retort stand and clamp. The inlet vessel was straightened to the required inlet length, as determined from eqn (2), to achieve laminar flow (Nicholas and O'Rourke 1990):

$$
\mathrm{L}=0.04 \mathrm{~d} \operatorname{Re}
$$

where: $\mathrm{L}(\mathrm{mm})$ is the inlet tube length required for laminar flow conditions, $\mathrm{d}(\mathrm{cm})$ is the vessel inner diameter and $\operatorname{Re}$ is the Reynolds number $(\operatorname{Re}=\rho d v / \eta)$, where $\rho$ is the fluid density, $\mathrm{v}\left(\mathrm{cm} \mathrm{s}^{-1}\right)$ is the highest mean velocity, $10 \mathrm{~cm} \mathrm{~s}^{-1}$ used in the experiment, and $\eta$ is the fluid viscosity. The minimum inlet lengths required to achieve laminar flow for the range of velocities used in the assessment for each vessel are listed in Table 2.

The mean velocity at which the BMF was pumped was initially set to $5 \mathrm{~cm} \mathrm{~s}^{-1}$. The vessel of interest was imaged longitudinally at the minimum depth (at the vessel inlet depth of $20 \mathrm{~mm}$ ) within the flow phantom in B-mode with the region-of-interest positioned in the centre of the field of view for each of the 10 curvilinear and six 
linear transducers and the image was optimised by adjusting the 2-D gain and the time-gain-controls, as well as positioning the focal zone at the depth of interest within the flow phantom.

The colour Doppler was enabled and the resulting colour Doppler image of the vessel was optimised by adjusting the size of the colour Doppler box, the gain and the position of focal zone to give the best possible image of the appropriate section of the vessel. The scanner's colour velocity setting, clutter filter setting and persistence setting were each set to their respective minimum values, in order for the lower velocities to be detected. The set mean velocity was decreased to the pump's lowest achievable velocity, listed in Table 3 for the three different size vessels, which was one of the limiting factors of the test, the second limiting factor being the depth of the vessel within the phantom. The colour Doppler gain was increased as far as possible without introducing noise and, if no velocity signal was detected, the pump velocity was increased until the scanner could detect the velocity signal; this velocity was recorded as the colour Doppler system's lowest detectable velocity with the vessel imaged at the minimum depth within the flow phantom $(20 \mathrm{~mm})$. Once the lowest detectable velocity was determined, the transducer was moved longitudinally along the length of the flow phantom, thus increasing the imaging depth. The penetration depth of the scanner was determined as the deepest point at which the colour Doppler signal could still be detected. The temperature of the flow phantom and the BMF was maintained at $22^{\circ} \mathrm{C} \pm 1{ }^{\circ} \mathrm{C}$, by controlling the room temperature.

The sensitivity performance index was then calculated from eqn. (1); this measurement was estimated to have an overall uncertainty of $\pm 4 \%(p>0.05)$. This 
was determined from the $95 \%$ confidence interval (CI) of the individual sources of systematic uncertainty and random uncertainty. The largest source of systematic uncertainty was the velocity accuracy for the flow phantom determined using timed weight collections of BMF. Both the resolution $(0.01 \mathrm{~g})$ of the weight collection and the resolution of the stop-watch $(0.017 \mathrm{~s})$ contributed to the velocity accuracy uncertainty of $0.5 \mathrm{~cm} \mathrm{~s}^{-1}$. The largest source of random uncertainty was the random error due to repositioning and reanalysis of the penetration depth $( \pm 4 \%)$, which was determined from repositioning the transducer five times, and the random error in the pumps set velocity $( \pm 2 \%)$, which was determined by five repeat timed weight collections of BMF over are range of pump velocities.

The criteria used to determine when colour/power Doppler reached the lowest detectable velocity and signal-to-noise threshold were determined by an experienced sonographer. The sonographer initially viewed a number of colour Doppler signals of decreasing velocity and then, once the lowest detectable velocity was determined, the velocity was set and the penetration depth was then determined in the $3.2 \mathrm{~mm}$ vessel of the flow phantom. The sonographer determined the appearance of the Doppler signal when it was of diagnostic quality, independently of knowing the lowest detectable velocity and the penetration depth used to produce the signal.

\section{Validation of sensitivity performance index}

Validation experiments were carried out to test the form of eqn (1) and to determine the optimum conditions for its use as a tool for the evaluation of colour Doppler sensitivity performance. The sensitivity performance index was validated by investigating the effect of vessel size on lowest detectable velocity, the relationship 
between vessel size and the sensitivity performance index and between mean velocity and the sensitivity performance index for the colour Doppler modes for a range of transducer and ultrasound scanner combinations.

The effect of vessel size on lowest detectable velocity was investigated by measuring the lowest detectable velocity in the three vessels (inner diameter $1.6 \mathrm{~mm}, 3.2 \mathrm{~mm}$ and $4.8 \mathrm{~mm}$ ) contained within the flow phantom, for the colour Doppler modes for the V4, L5 and L738 transducers (128), the 4C1, L5 and L7 transducer (Aspen) and the C5-2, L7-4 and L12-5 transducers (HDI 5000). The relationship between 1/vessel-size and the sensitivity performance index was investigated by measuring the penetration depth in the three vessels for the colour Doppler modes for the V4 transducer (128), the $4 \mathrm{C} 1$ transducer (Aspen) and the C5-2 transducer (HDI 5000), for the lowest detectable velocity and, thus, calculating the sensitivity performance index.

The relationship between 1/mean-velocity and the sensitivity performance index was investigated by measuring the penetration depth for the velocity range of $1-5 \mathrm{~cm} \mathrm{~s}^{-1}$ for the colour Doppler modes for the 4C1 transducer (Aspen) and the CX5-2 transducer (Antares). The relationship between mean velocity and the sensitivity performance index was also investigated for each of the three vessels of different inner diameter.

The sensitivity performance index for the validation experiments was determined using the test protocol described in the previous section. 


\section{The effect of instrument settings on the sensitivity performance index}

The effect of instrument settings on the sensitivity performance index were investigated in order to determine whether they had an effect on the sensitivity performance index and, if so, to identify the optimum instrument settings.

Transducer frequency. The effect of transducer type and frequency on the sensitivity performance index was investigated for the 4C1, L5 and L7 transducers (Aspen), the V4, L5 and L738 transducers (128) and the C5-2, L7-4 and L12-5 transducers (HDI 5000).

Clutter filter setting. The effect of clutter filter setting on the sensitivity performance index was investigated for the 4C1 transducer (Aspen) and the PVM375AT transducer (Nemio), a high-range and a mid-range system. The other colour instrument settings, including colour gain, output power, colour persistence and focal zones, were fixed while the clutter filter setting was changed.

Output power. The effect of output power on the sensitivity performance index was investigated for the 4C1 transducer (Aspen) and the PVM375AT transducer (Nemio). The other colour instrument settings, including colour gain, clutter filter setting, colour persistence and focal zones, were fixed while the output power was changed.

Persistence setting. The effect of persistence on the sensitivity performance index was investigated for the $4 \mathrm{C} 1$ transducer (Aspen) and the PVM375AT transducer (Nemio). The other colour instrument settings, including colour gain, clutter filter setting, output power and focal zones, were fixed while the colour persistence was changed.

The sensitivity performance index for the four experiments was determined using the previously described test protocol. 
Evaluation of the sensitivity performance index for a range of ultrasound scanners

Ten curvilinear transducers were tested with the "abdominal" colour Doppler application settings for imaging the abdomen, while six linear transducers were tested with the "vascular" colour Doppler application settings for imaging the carotid artery, using the sensitivity performance index test protocol. This process was repeated for the power Doppler mode.

\section{RESULTS}

\section{Validation of sensitivity performance index}

It was found that the lowest detectable velocity was strongly influenced by the inner diameter of the vessel (Table 5). A decrease in the diameter size resulted in a decrease in the colour Doppler signal strength; therefore, the limiting factor for the detection of the lowest velocity was found to be the vessel inner diameter.

A nonlinear relationship was found between $1 /$ vessel-size and sensitivity performance index for each of the general-purpose abdominal transducers of the three ultrasound scanners (Fig. 2). In general, it was found that the $3.2 \mathrm{~mm}$ inner diameter vessel demonstrated the clearest differentiation between the general purpose abdominal transducers of the three ultrasound scanners, whereas, for the $1.6 \mathrm{~mm}$ and $4.8 \mathrm{~mm}$ inner diameter vessels, the transducers had similar sensitivity performance indices (Fig. 2). 
A linear relationship was found between 1 /velocity and sensitivity performance index for the three vessels sizes with the 4C1 transducer (Aspen) (Fig. 3a) as well as for the three vessel sizes with the CX5-2 transducer (Antares) (Fig. 3b).

\section{The effect of instrument settings on the sensitivity performance index}

The effects of the instrument settings are shown in Figs. 4 to 7 . It was found that these had a marked effect on the sensitivity performance index: increasing the nominal frequency and the clutter filter setting caused the index to decrease, while increasing output power and colour persistence setting improved the index score.

The sensitivity performance index of the colour Doppler for the 4C1, L5 and L7 transducers (Aspen), the V4, L5 and L738 transducer (128) and the C5-2, L7-4 and L12-5 transducers (HDI 5000) decreased with increasing frequency and transducer type (Fig. 4).

The sensitivity performance index of the colour Doppler for the $4 \mathrm{C} 1$ transducer (Aspen) and the PVM375AT transducer (Nemio) decreased with increasing clutter filter setting with a change of clutter filter setting from 3 to 4 and from 4 to 5 , respectively, having the most significant effect on the sensitivity performance index, as illustrated in Figs. 5a and 5b.

The sensitivity performance index for the colour Doppler of the $4 \mathrm{C} 1$ transducer (Aspen) and the PVM375AT transducer (Nemio) increased with increasing output power, as illustrated in Fig. 6. 
The sensitivity performance index of the colour Doppler for the $4 \mathrm{C} 1$ transducer (Aspen) was highest for the medium- and high-persistence setting; however, the effect of persistence on the sensitivity performance index was small, while the effect of increasing persistence for the PVM375AT transducer (Nemio) caused a significant improvement in sensitivity performance, as illustrated in Fig. 7.

\section{Evaluation of the sensitivity performance index for a range of}

\section{ultrasound scanners}

It was found that the curvilinear transducers of the Aplio and the Antares scanners achieved the highest sensitivity performance index, while the 128 scanner had the lowest, as illustrated in Fig. 8. Among the vascular transducers, the Antares and the HDI 5000 scanners achieved the highest sensitivity performance index, while the 128 scanner had the lowest, as illustrated in Fig. 9. The sensitivity performance index results were as expected for the different scanners when correlating the results with the cost of the scanner $\left(\mathrm{R}^{2}=0.86, \mathrm{p}>0.002\right)$, which is reflective of the complexity of the scanner; for example, the newest high-range scanners had the best performance while the oldest scanner, despite being high-range 12 years ago, had the worst sensitivity performance. The sensitivity performance index appeared to show good differentiation between the different scanners, reflecting their complexity. 


\section{DISCUSSION}

Doppler sensitivity is an important aspect of Doppler performance, since, in day-today clinical examinations, the question often arises as to whether blood flow is present. It is clinically important to distinguish between the presence of trickle blood flow and no blood flow, in order for the type of patient management to be decided; for example, this decides between treatment with medication or surgery for a carotid artery stenosis. The sensitivity of the colour and power Doppler modes is influenced by two factors, the detection of low velocity and low volume blood flow (Hoskins et al 1994; Wang et al 1992).

The sensitivity performance index was validated by investigating the relationship between vessel size and lowest detectable velocity and between both vessel size and mean velocity with the sensitivity performance index. The lowest detectable velocity was affected by the inner diameter of the vessel and, therefore, the volume blood flow: the $3.2 \mathrm{~mm}$ and $4.8 \mathrm{~mm}$ inner diameter vessels demonstrated similar results and showed clear differences between the different ultrasound scanners, while the $1.6 \mathrm{~mm}$ inner diameter vessel proved to be more challenging and showed no differences between the different ultrasound scanners. Furthermore, the three different frequency transducers of the 128, Aspen and HDI 5000 scanners did not detect different lowest detectable velocities: it was expected that the higher frequencies of the linear transducers would detect a lower velocity. This result may be due to the clutter filter cut-off frequency, which was found to affect the results of the Aspen and the Nemio ultrasound scanners (Figs. 5a and 5b), indicating that the clutter filter was the next limiting factor for lowest detectable velocity after the volume of blood flow (Table 4). It was found that the relationship between the sensitivity performance index and 
vessel size was not proportional, but this can be approximately accounted for by considering the effect of the acoustic properties of the vessel material, the clutter filter and beam shape (Deverson and Evans 2000). The speed of sound of the c-flex tubing $\left(1553 \mathrm{~m} \mathrm{~s}^{-1}\right)$ is greater than those of the surrounding TMM $\left(1540 \mathrm{~m} \mathrm{~s}^{-1}\right)$ and the BMF $\left(1548 \mathrm{~m} \mathrm{~s}^{-1}\right)$, which leads to total internal reflection at the interface between the vessel and the surrounding medium and refraction of the incident sound beam (Hoskins et al 1994). Furthermore, there will also be differential attenuation of the beam at the different points, due to different path lengths within the vessel. Therefore, the acoustic properties of the vessel material may have had an effect on the detected colour Doppler signal by reducing the low frequency components of the signal (Thompson et al 2000). High-pass filtering, which is used in Doppler equipment to remove low-frequency high amplitude clutter signals that occur due to slow-moving tissue in the path of the beam, will, in addition to removing clutter signals, also remove the low-frequency signal originating from the low-velocity red blood cells travelling close to the vessel walls (Evans and McDicken 2000). Furthermore, nonuniform insonation of the vessel would result in unequal weighting of the colour Doppler signal at different frequencies, depending on the intensity of the beam at the position in the vessel from which the each signal originates (Deverson and Evans 2000). The results obtained using flow phantoms with vessel sizes different to those used in this study are, therefore, not directly comparable with the results found in this study however, a similar trend should be found in the results for the effect of control settings and a similar range of ultrasound scanners. In general, it was found that the $3.2 \mathrm{~mm}$ inner diameter vessel demonstrated the clearest differentiation between the general purpose abdominal transducers of the three ultrasound scanners, whereas, for the $1.6 \mathrm{~mm}$ and $4.8 \mathrm{~mm}$ inner diameter vessels, the transducers had similar sensitivity 
performance indices (Fig. 2). Therefore, the $3.2 \mathrm{~mm}$ inner diameter vessel was used to determine the sensitivity performance index throughout the remainder of this study.

A linear relationship was found between 1 /velocity and sensitivity performance index for the three vessels sizes with the 4C1 transducer (Aspen) and the CX5-2 transducer (Antares), which was expected since, the lower the velocity detected, the higher was the sensitivity index.

The effect of instrument setting, such as nominal Doppler frequency and transducer type, clutter filter, output power and persistence setting, on the sensitivity performance index were investigated and the optimum settings were identified; this is especially important as ultrasound scanners reach new degrees of sophistication and many parameters are available for user control and selection. The nominal frequency and transducer type, clutter filter and output power were found to have the most significant effect on the sensitivity performance index of the respective ultrasound scanners; increasing the nominal frequency or the clutter filter setting caused a decrease in sensitivity performance, while increasing the output power caused an increase in sensitivity performance. Wang et al (1992) found similar trends in sensitivity performance with the increase of the clutter filter and output power settings. The different persistence settings were found to have no effect on the $4 \mathrm{C} 3$ transducer (Aspen), whereas increasing the persistence setting of the PVM375AT transducer (Nemio) was found significantly to improve its sensitivity performance. Therefore, clinicians need to be aware of the effect of these settings on the colour Doppler sensitivity performance of their ultrasound scanner when interpreting the clinical significance of the colour Doppler information. In particular, these effects are 
especially important when evaluating serial examinations for changes in the character of blood flow.

The sensitivity performance index used in this paper is a figure of merit and was developed to give a combined measure of related aspects of sensitivity, such as the lowest detectable velocity, the vessel size and the penetration depth. The sensitivity performance index scores were found to demonstrate a good correlation with the cost $\left(\mathrm{R}^{2}=0.86, \mathrm{p}>0.002\right)$ of the ultrasound scanners. It was found that the Antares and the HDI 5000 scanners, both high-end systems, had the highest index scores for both the curvilinear and linear transducers. The sensitivity index values were, in general, higher for the curvilinear transducer than for the linear transducer, due to the higher penetration depth values for the curvilinear transducers.

There have been attempts previously by Wang et al (1992) and Rickey and Fenster (1996) to determine colour Doppler sensitivity performance. The attempt made by Wang et al (1992) was unsuccessful in determining colour Doppler sensitivity, due to the presence of bidirectional symmetrical side-bands. Rickey and Fenster (1996) used a complex test phantom which has not been replicated to date and the generation of a 4-D data-set makes the analysis time-consuming and not practical for quality control tests in a busy ultrasound department. The sensitivity performance index evaluated in this paper to measure colour Doppler sensitivity is simple and quick to use, ideal for quality control testing in a busy ultrasound department. This sensitivity performance index may also be used to determine spectral Doppler sensitivity performance and has been found to show good differentiation between scanners of varying complexity and age (Browne et al 2002). 


\section{CONCULSIONS}

In this paper, a Doppler sensitivity performance index was described and validated, with which colour and power Doppler sensitivity may be evaluated quickly and easily. It was found that the relationship between vessel size and the sensitivity performance index was not proportional, but may be accounted for by considering the effect of the acoustic properties of the vessel material, the clutter filter and beam shape. Use of the diameter parameter in the index should enable the technique to be used with alternative flow phantoms with different vessel sizes. The results from such flow phantoms would not be directly comparable with the results found in this study; however, a similar trend should be found in the results for the effect of control settings and a similar range of ultrasound scanners.

Furthermore, using the sensitivity performance index demonstrated that differences between scanners of varying complexity and age could be identified, in addition to being sensitive to differences between scanners of similar complexity. The sensitivity performance index described can be used to test and quantify the sensitivity of the ultrasound scanner for different combinations of instrument settings. The preliminary data collected demonstrate that curvilinear transducers have higher sensitivity performance indices than linear transducers, due to the higher achievable penetration depth. Furthermore, the colour Doppler sensitivity performance was found to be significantly dependent on the nominal frequency and transducer type, clutter filter setting and the output power setting. 


\section{REFERENCES}

AIUM. Performance criteria and measurements for Doppler ultrasound devices. American Institute of Ultrasound in Medicine Standards Committee. 1993;

Allan PL, Dubbins PA, Pozniak MA, and McDicken WN. Clinical Doppler Ultrasound. 2000;123-190.

Baxter GM. The role of ultrasound in deep vein thrombosis. Clinical Radiology. 1997;52:1-3.

Browne JE, Watson AJ, Hoskins PR, and Thomson C. Evaluation of a Doppler sensitivity phantom. European Journal of Ultrasound. 2002;15:S15-

Deverson S and Evans DH. Using Doppler signal power to detect changes in vessel size: A feasibility study using a wall-less flow phantom. Ultrasound in Medicine and Biology. 2000;26:593-602.

Evans DH and McDicken WN. Doppler Ultrasound: Physics, instrumentation and signal processing. 2000;229-282.

Hoskins PR, Sherriff SB, and Evans JA. Testing Doppler Ultrasound Equipment. 1994;

Martin K and Spinks D. Measurement of the speed of sound in ethanol/water mixtures. Ultrasound in Medicine and Biology. 2001;27:289-291.

Mattos MA, Hodgson KJ, Ramsey DE, Barkmeier LD, and Summer DS. Identifying total carotid occulsion with colour flow duplex scanning. European Journal of Vascular Surgery. 1992;6:204-210.

Nicholas WW and O'Rourke MF. McDonalds blood flow in arteries, theoretical, experimental and clinical principles. 1990; 
Ramnarine KV, Hoskins PR, Routh HF, and Davidson F. Doppler backscatter properties of a blood-mimicking fluid for Doppler performance assessment. Ultrasound in Medicine and Biology. 1999;25:105-110.

Ramnarine KV, Nassiri DK, Hoskins PR, and Lubbers J. Validation of a new bloodmimicking fluid for use in Doppler flow test objects. Ultrasound in Medicine and Biology. 1998;24:451-459.

Rickey DW and Fenster A. A Doppler ultrasound clutter phantom. Ultrasound in Medicine and Biology. 1996;22:747-766.

Thompson RS, Tortoli P, and Aldis GK. Selective transmission of a focused Doppler ultrasound beam through a plastic layer. Ultrasound in Medicine and Biology. 2000;26:1333-1346.

Wang KY, Bone SN, and Hossack JM. A tool for evaluating Doppler sensivitivity. The Journal of Vascular Technology. 1992;16:87-94. 
Table 1. Physical and acoustic properties of the blood-mimicking fluid used compared with whole human blood.

\begin{tabular}{|c|c|c|}
\hline Properties & $\begin{array}{c}\text { Human blood } \\
\left(37^{\circ} \mathrm{C}\right)\end{array}$ & $\begin{array}{l}\text { Blood-mimicking } \\
\text { fluid }\left(22^{\circ} \mathrm{C}\right)\end{array}$ \\
\hline Scatterer & Red blood cells & Orgasol $^{\mathrm{TM}}$ (nylon) \\
\hline Scatterer size $(\mu \mathrm{m})$ & 7 & 5 \\
\hline Hematocrit (\% volume) & 45 &, 5 \\
\hline Density $\left(\mathrm{kg} \mathrm{m}^{-3}\right)$ & 1053 & $1037 \pm 2$ \\
\hline Viscosity (mPa s) & 3 & $4.1 \pm 0.1$ \\
\hline Velocity $\left(\mathrm{m} \mathrm{s}^{-1}\right)$ & 1583 & $1548 \pm 5$ \\
\hline $\begin{array}{c}\text { Attenuation } \\
\left(\mathrm{dB} \mathrm{cm}^{-1} \mathrm{MHz}^{-1}\right)\end{array}$ & 0.15 & $0.05 \pm 0.01$ \\
\hline Backscatter $\left(\mathrm{f}^{4} \mathrm{~m}^{-1} \mathrm{sr}^{-1}\right)$ & $4 \times 10^{-31}$ & $\begin{array}{l}\text { Continuous flow } \\
\text { human blood }\end{array}$ \\
\hline Fluid properties & Non-Newtonian & Newtonian \\
\hline
\end{tabular}

Table 2. Flow phantom inlet lengths (for maximum velocity $=10 \mathrm{~cm} \mathrm{~s}^{-1}$ ).

\begin{tabular}{cc}
\hline \hline Diameter of vessel $(\mathbf{c m})$ & Inlet length (cm) \\
\hline \hline 0.16 & 0.1 \\
0.32 & 0.5 \\
0.48 & 1.2 \\
\hline \hline
\end{tabular}

Table 3. Lowest achievable velocities in the three vessels of the flow phantom.

\begin{tabular}{cccc}
\hline \hline Vessel diameter & $\mathbf{1 . 6} \mathbf{~ m m}$ & $\mathbf{3 . 2} \mathbf{~ m m}$ & $\mathbf{4 . 8} \mathbf{~ m m}$ \\
\hline \hline $\begin{array}{c}\text { Lowest achievable } \\
\text { velocity }\left(\mathrm{cm} \mathrm{s}^{-1}\right)\end{array}$ & 0.6 & 0.8 & 0.8 \\
\hline \hline
\end{tabular}


Table 4. Summary of ultrasound scanners used with associated transducers and modes tested.

\begin{tabular}{|c|c|c|c|c|}
\hline $\begin{array}{l}\text { Ultrasound } \\
\text { scanner }\end{array}$ & $\begin{array}{c}\text { Transducer } \\
\text { (colour Doppler } \\
\text { frequency) }\end{array}$ & Modes Tested & $\begin{array}{l}\text { Approximate } \\
\text { cost- when } \\
\text { purchased }\end{array}$ & Age \\
\hline Acuson 128 & $\begin{array}{l}\text { V4 (2.5 MHz) } \\
\text { L5 (3.5 MHz) } \\
\text { L738 (5 MHz) }\end{array}$ & Colour Doppler & $£ 40000$ & $12 \mathrm{y}$ \\
\hline $\begin{array}{l}\text { Acuson } \\
\text { Aspen }\end{array}$ & $\begin{array}{c}\text { 4C1 }(2.5 \mathrm{MHz}) \\
\text { L5 (3.5 MHz) } \\
\text { L7 (5 MHz) }\end{array}$ & $\begin{array}{l}\text { Colour Doppler } \\
\text { Power Doppler }\end{array}$ & $£ 80000$ & $4 y$ \\
\hline $\begin{array}{c}\text { ATL HDI } \\
5000\end{array}$ & $\begin{array}{c}\mathrm{C} 5-2(2.5 \mathrm{MHz}) \\
\mathrm{L} 7-4(4 \mathrm{MHz}) \\
\text { L12-5 (6 MHz) }\end{array}$ & $\begin{array}{l}\text { Colour Doppler } \\
\text { Power Doppler }\end{array}$ & $£ 100000$ & $3 y$ \\
\hline $\begin{array}{c}\text { Aloka SSD } \\
5000\end{array}$ & $\begin{array}{l}\text { UST-9119 (5 } \\
\text { MHz) }\end{array}$ & $\begin{array}{l}\text { Colour Doppler } \\
\text { Power Doppler }\end{array}$ & $£ 80000$ & 2 months \\
\hline $\begin{array}{l}\text { Siemens } \\
\text { Sienna }\end{array}$ & $\mathrm{C} 5-2(2-5 \mathrm{MHz})$ & $\begin{array}{l}\text { Colour Doppler } \\
\text { Power Doppler }\end{array}$ & $£ 45000$ & 3 months \\
\hline $\begin{array}{c}\text { SonoSite } \\
180\end{array}$ & $\mathrm{C} 5-2(2-5 \mathrm{MHz})$ & $\begin{array}{c}\text { Directional } \\
\text { Power Doppler } \\
\text { Power Doppler }\end{array}$ & $£ 25000$ & $1 \mathrm{y}$ \\
\hline $\begin{array}{l}\text { SonoSite } \\
\text { 180Plus }\end{array}$ & $\mathrm{C} 5-2(2-5 \mathrm{MHz})$ & $\begin{array}{c}\text { Directional } \\
\text { Power Doppler } \\
\text { Power Doppler }\end{array}$ & $£ 20000$ & 1 month \\
\hline $\begin{array}{l}\text { Toshiba } \\
\text { Nemio }\end{array}$ & $\begin{array}{l}\text { PVM375AT } \\
(4 \mathrm{MHz}) \\
\text { PLM703AT } \\
(7 \mathrm{MHz})\end{array}$ & $\begin{array}{l}\text { Colour Doppler } \\
\text { Power Doppler }\end{array}$ & $£ 70000$ & $<1 \mathrm{y}$ \\
\hline $\begin{array}{c}\text { Toshiba } \\
\text { Aplio }\end{array}$ & $\begin{array}{l}\text { PVT375AT } \\
(4 \mathrm{MHz}) \\
\text { PLT703AT } \\
(7 \mathrm{MHz}) \\
\end{array}$ & $\begin{array}{l}\text { Colour Doppler } \\
\text { Power Doppler }\end{array}$ & $£ 120000$ & $<1 \mathrm{y}$ \\
\hline $\begin{array}{l}\text { Siemens } \\
\text { Antares }\end{array}$ & $\begin{array}{l}\text { CX5-2 (4 MHz) } \\
\text { VX9-4 }(7 \mathrm{MHz})\end{array}$ & $\begin{array}{l}\text { Colour Doppler } \\
\text { Power Doppler }\end{array}$ & $£ 120000$ & $<1 \mathrm{y}$ \\
\hline
\end{tabular}


Table 5. Effect of vessel size on the lowest detectable velocity for three ultrasound scanners of varying complexity.

\begin{tabular}{|c|c|c|c|}
\hline $\begin{array}{l}\text { Lowest detectable } \\
\text { velocity }\left(\mathrm{cm} \mathrm{s}^{-1}\right)\end{array}$ & $\begin{array}{c}1.6 \mathrm{~mm} \text { inner } \\
\text { diameter vessel } \\
\left(\mathrm{cm} \mathrm{s}^{-1}\right)\end{array}$ & $\begin{array}{c}3.2 \mathrm{~mm} \text { inner } \\
\text { diameter vessel } \\
\left(\mathrm{cm} \mathrm{s}^{-1}\right)\end{array}$ & $\begin{array}{l}4.8 \mathrm{~mm} \text { inner } \\
\text { diameter vessel } \\
\left(\mathrm{cm} \mathrm{s}^{-1}\right)\end{array}$ \\
\hline Acuson 128 (V4) & 3.3 & 1.7 & 1.6 \\
\hline Acuson 128 (L5) & 3.3 & 1.7 & 1.6 \\
\hline Acuson 128 (L738) & 3.3 & 1.7 & 1.6 \\
\hline Acuson Aspen (4C1) & 3.3 & 1.7 & 1.6 \\
\hline Acuson Aspen (L5) & 3.3 & 1.7 & 1.6 \\
\hline Acuson Aspen (L7) & 3.3 & 1.7 & 1.6 \\
\hline ATL HDI 5000 (C5-2) & 3.3 & 1 & 0.8 \\
\hline ATL HDI 5000 (L7-4) & 3.3 & 1 & 0.8 \\
\hline ATL HDI 5000 (L12-5) & 3.3 & 1 & 0.8 \\
\hline
\end{tabular}


Fig. 1. Schematic of the penetration depth flow phantom set-up.

Fig. 2. Effect of 1/vessel size on sensitivity performance index

Fig. 3.a. Effect of 1/velocity on sensitivity performance index using the $4 \mathrm{C} 1$ transducer of the Acuson Aspen b. Effect of 1/velocity on sensitivity performance index using the CX5-2 transducer of the Siemens Antares.

Fig. 4. Effect of transducer and colour Doppler frequency on the sensitivity performance index of 4C1, L5, L7 transducers (Aspen), V4, L5, L738 transducers (128) and C5-2, L7-4, L12-5 transducers (HDI 5000).

Fig. 5a. Effect of clutter filter settings on the sensitivity performance index of the $4 \mathrm{C} 1$ transducer (Aspen) b. Effect of clutter filter settings on the sensitivity performance index of the PVM375AT transducer (Nemio).

Fig. 6. Effect of output power on the sensitivity performance index of $4 \mathrm{C} 1$ transducer (Aspen) and PVM375AT transducer (Nemio).

Fig. 7. Effect of colour persistence on the sensitivity performance index of $4 \mathrm{C} 1$ transducer (Aspen) and PVM375AT transducer (Nemio).

Fig. 8. Sensitivity performance index of colour and power Doppler for the general purpose curvilinear transducers of the 10 ultrasound scanners.

Fig. 9. Sensitivity performance index of colour and power Doppler for the vascular linear transducers of the six ultrasound scanners. 


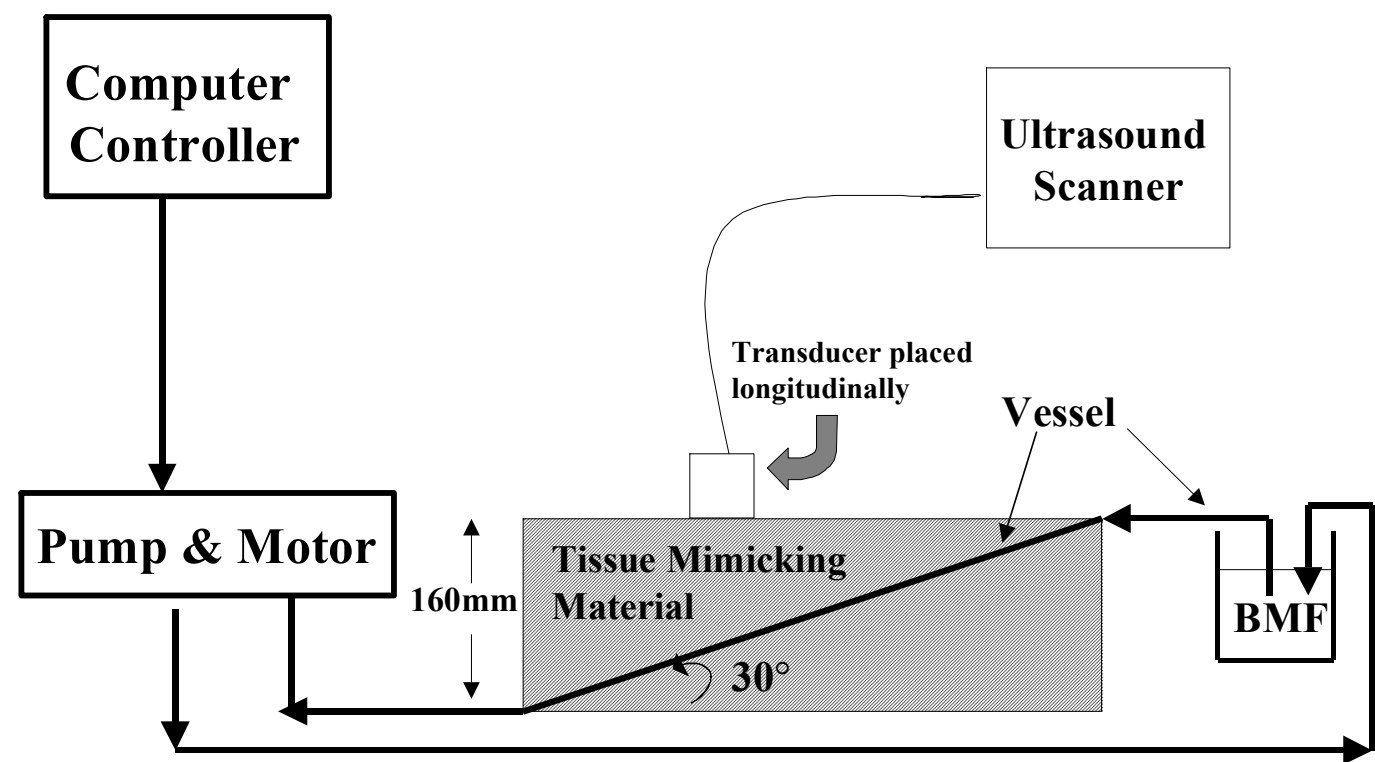

Direction of Flow 


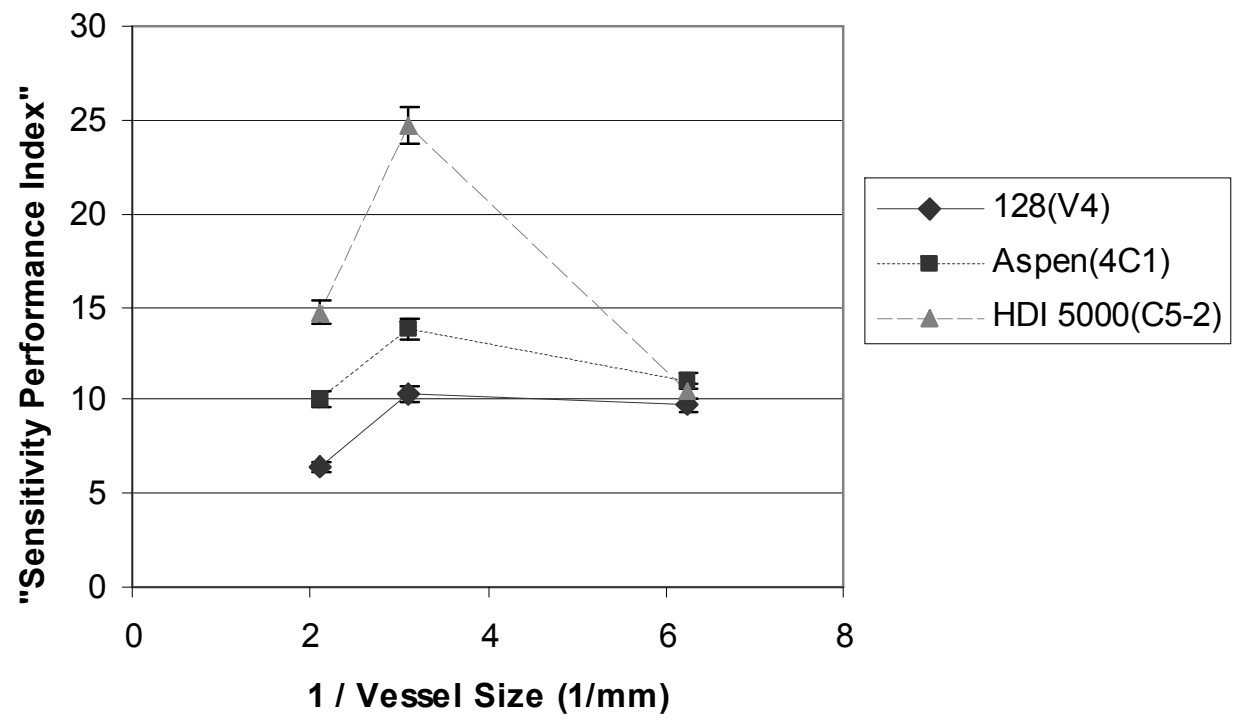









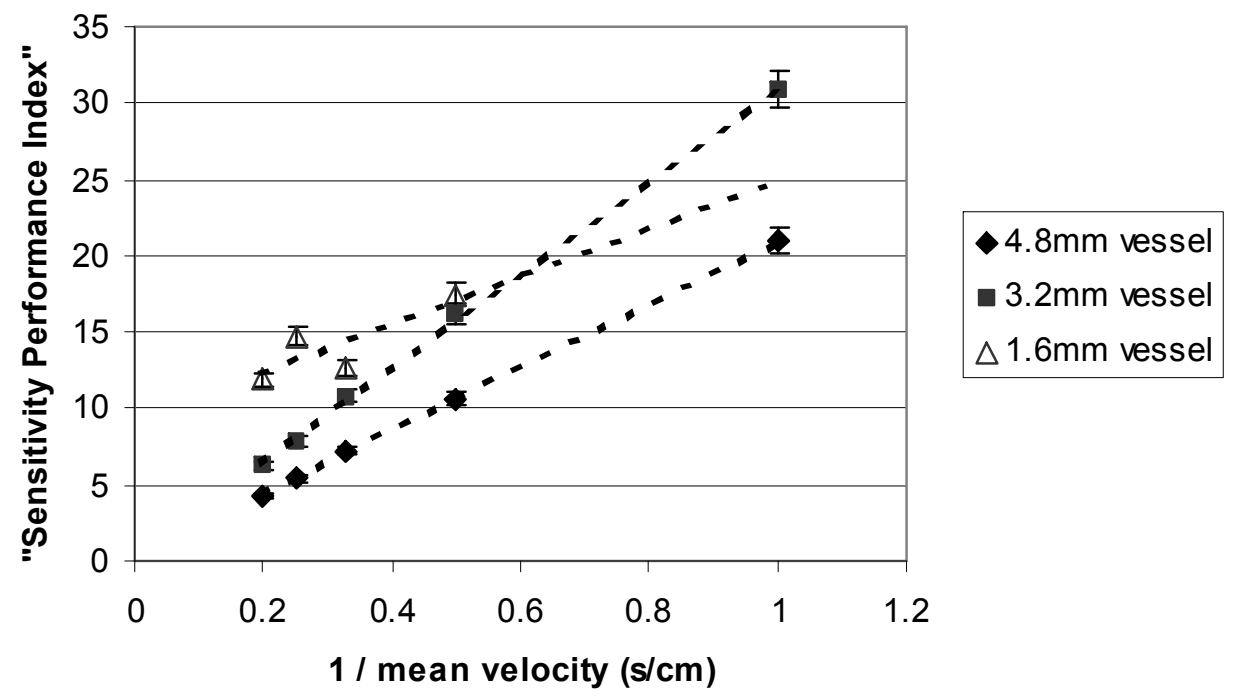




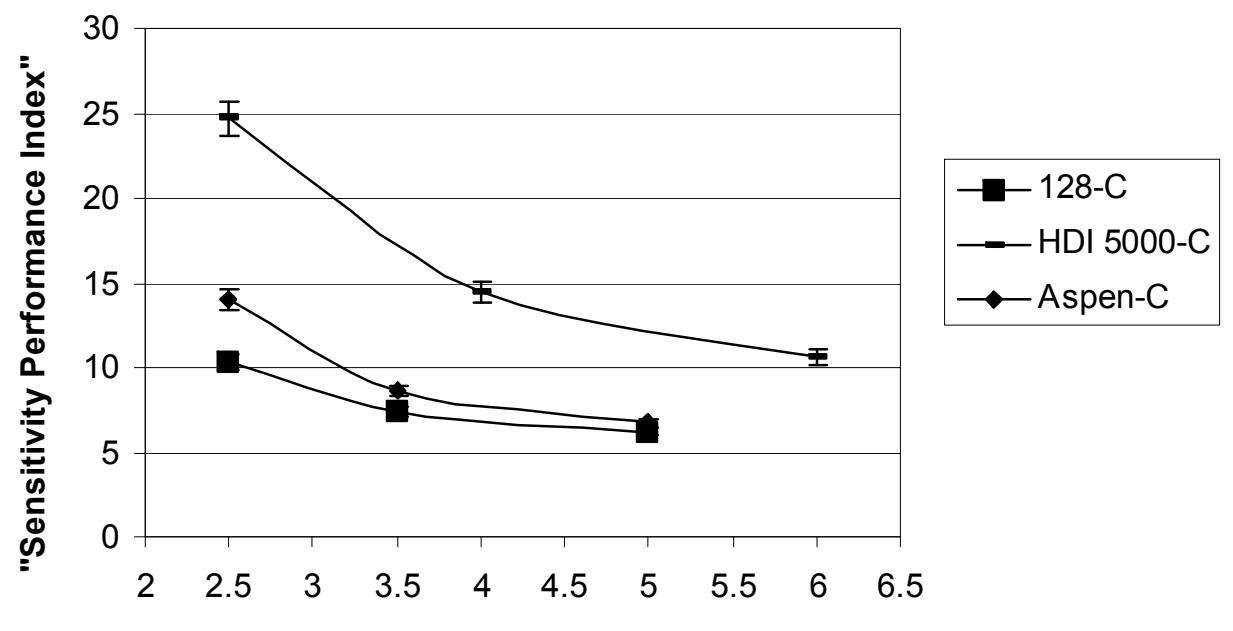

Nominal Colour Doppler Frequency $(\mathrm{MHz})$ 


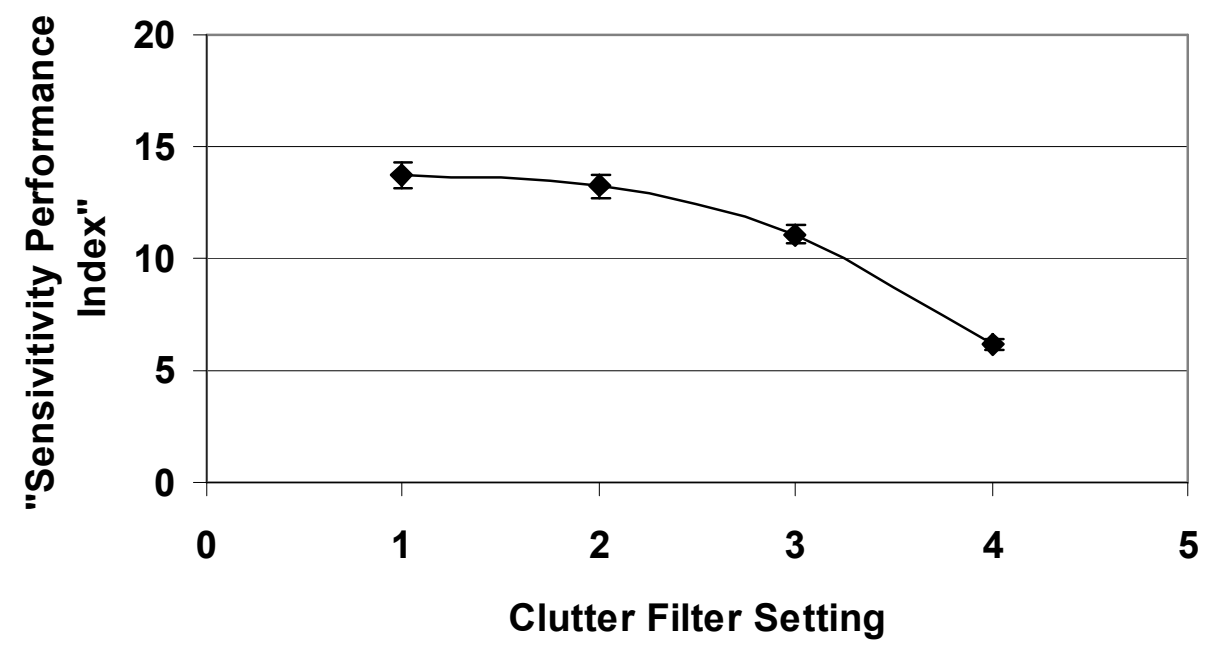




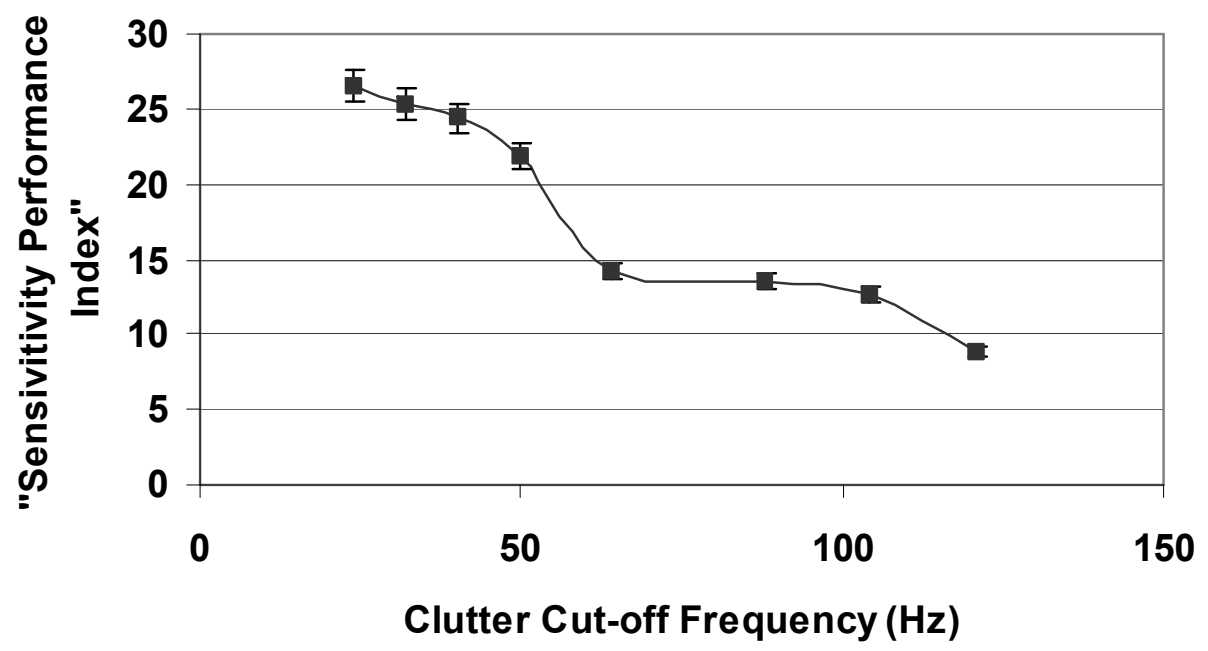




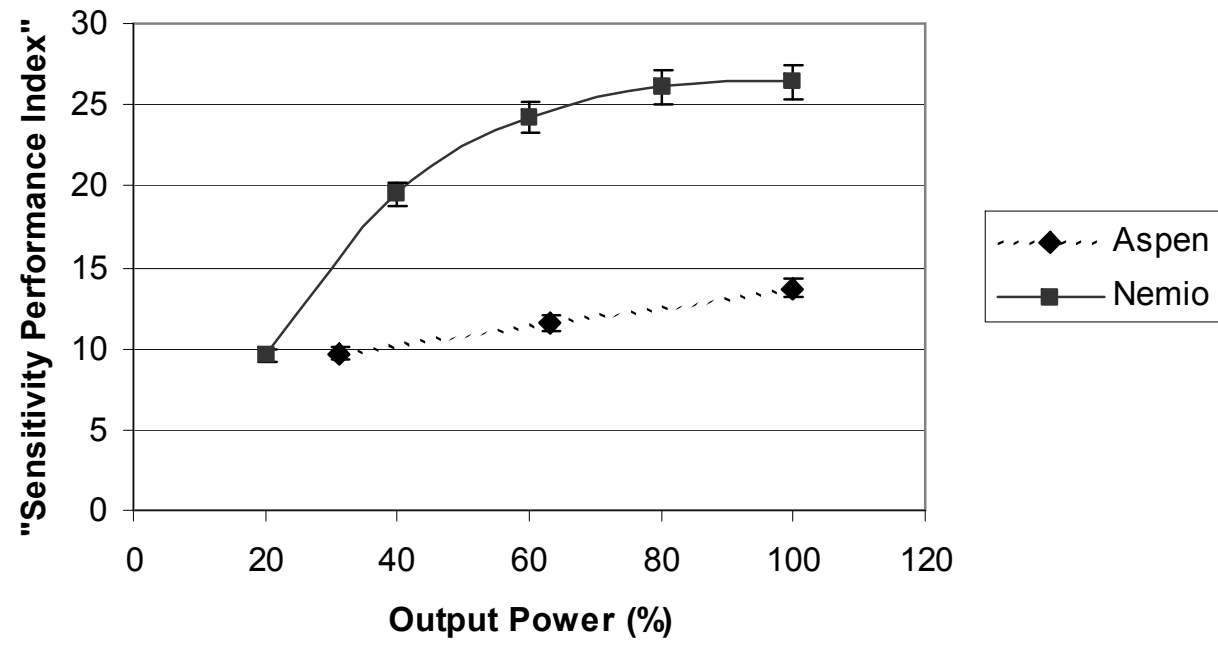




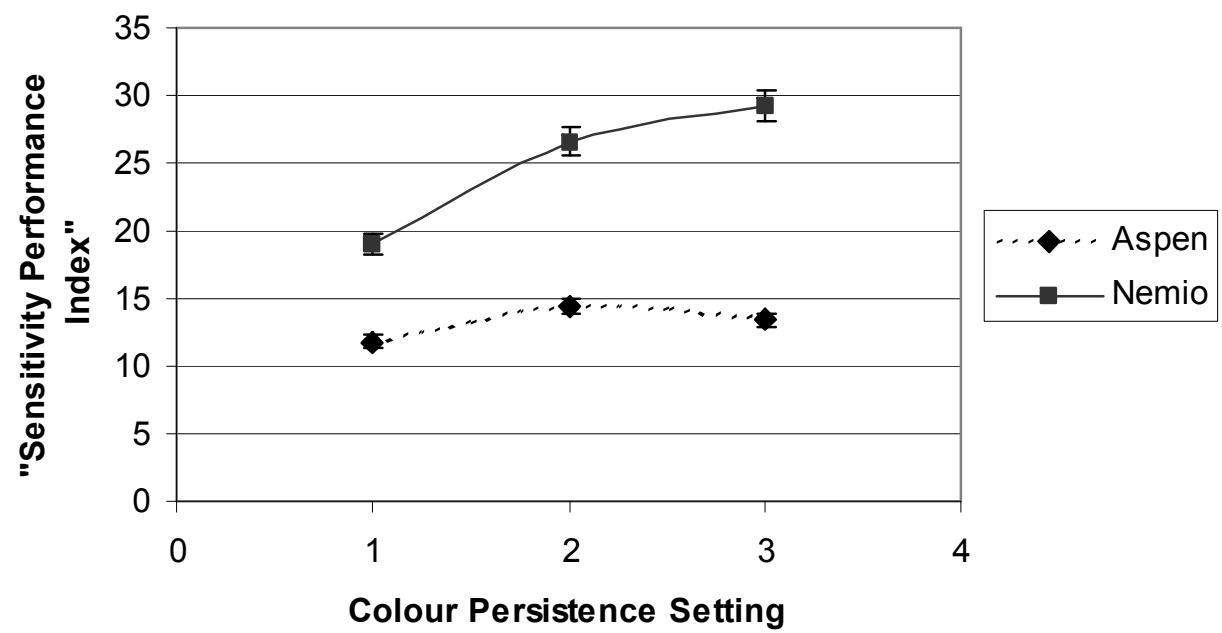




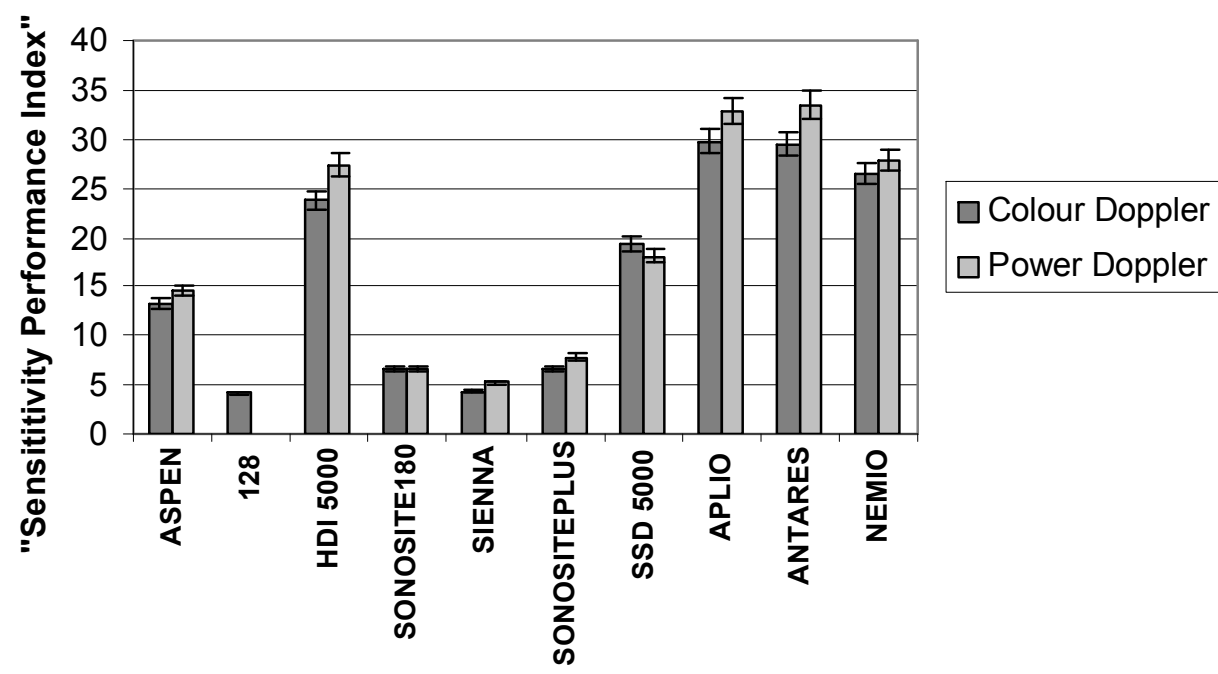




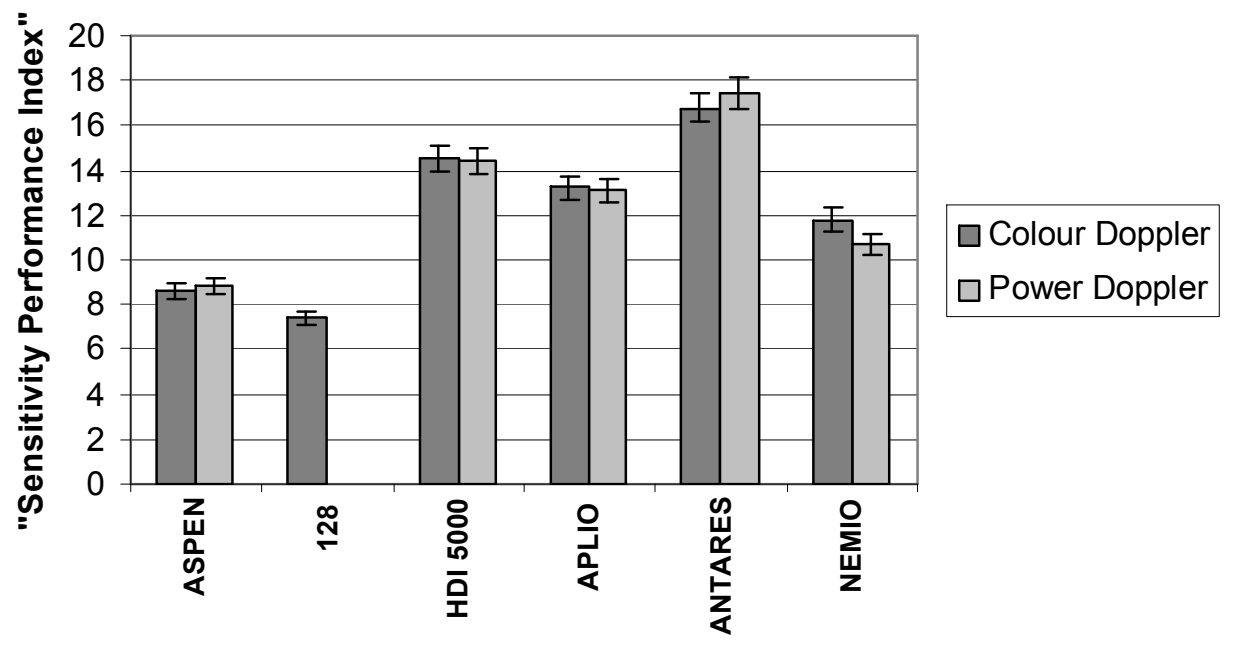

